\title{
Neurological aspects that should not be forgotten during the COVID-19 pandemic
}

Luis E. Fernández-Garza'

Alejandro Marfil ${ }^{1}$

In December 2019, an outbreak of a novel coronavirus causing severe acute respiratory distress (later named Severe Acute Respiratory Syndrome by a Coronavirus 2 (SARS-CoV-2)) emerged in Wuhan, China, and then rapidly spread throughout the world, becoming the worst 21 st-century pandemic so far. At the beginning of April 2020, a million confirmed cases and $>60,000$ deaths were reached around the world, with a constant increase that, at the present day, we do not know when it will be controlled. Recently, the World Health Organization called the disease caused by SARS-CoV2 as coronavirus disease 2019 (COVID-19).

In addition to the respiratory and systemic symptoms most frequently presented in COVID-19, there are some neurological symptoms reported. Around 36\% of patients have had neurological manifestations that involved the central nervous system (dizziness, headache, altered mental status, ataxia, and seizures), peripheral nervous system (anosmia, ageusia, and neuralgia), and musculoskeletal system (fatigue, twitching, and myalgias). These have been more frequent in patients with severe COVID-19. The lymphocyte count seems to be lower in patients with neurological symptoms. This phenomenon may be indicative that the weaker the response of the immune system, the greater the neurological risk of involvement $[1,2]$.

The impairment of taste and smell are two identified symptoms that are present in patients with nonsevere illness and maybe decisive to lower the transmission of this virus. Some reports inform prevalence of $5 \%$ $66 \%[3,4]$. Two possible forms of virus invasion have been discussed due to its similarity with SARS-CoV. SARS-CoV has been found in the brain and, more abundantly, in the brainstem of patients and experimental models, for that reason it is thought that SARS-COV2 is a neurotropic virus that infects cells through interactions between its spike protein (S1) and the Angiotensin-Converting Enzyme 2 (ACE2) receptor, and the help of the cell surface protease TMPRSS2 [5]. It has been demonstrated that the olfactory sensory neurons do not express these two receptors, but the olfactory epithelial support cells and the nasal respiratory epithelium do it widely, giving an open path to the olfactory bulb, via the cribriform plate. The second hypothesis is that direct damage to the olfactory and gustatory receptors by the SARS-CoV2 is possible [6].

The worst clinical manifestation of COVID-19 infection is respiratory distress, and it is the most frequent reason for admission to an intensive care unit. If we follow the premise of the neural invasion of SARS-CoV2, it is believed that SARS-CoV2 could spread via synapses from the mechano and chemoreceptors in the lung and lower respiratory system to infect neurons of the nuclei associated with cardio-respiratory control, and that could partially be responsible for the respiratory failure, the main cause of death of COVID-19 patients [7].

Severe cases of COVID-19 can have acute neurological complications. Among them, stroke, both hemorrhagic and ischemic, is the most frequently reported. Multiple

\footnotetext{
${ }^{1}$ Neurology Department, University Hospital "Dr. José Eleuterio González", Autonomous University of Nuevo Leon. Correspondence to: LE FERNÁNDEZ-GARZA. E-mail: <luisfdz12@gmail.com>.

$\otimes \square \otimes \square \square$
} 
factors can contribute. SARS-CoV2 also binds ACE2 receptors in the vascular endothelial cells. In patients with previous systemic hypertension, it may cause fluctuations in blood pressure that will increase the risk of intracranial hemorrhage. Also, COVID-19 patients often show elevated levels of D-dimer and severe thrombocytopenia, which can contribute to embolic vascular events and intracerebral bleeding, respectively [1]. There is one report of three COVID-19 patients with coagulopathy, antiphospholipid antibodies, and multiple cerebral infarcts, that suggest the induction of an antiphospholipid syndrome through viral infection [8].

There are reports of isolated cases of other possible neurological complications of COVID-19. One patient with seizures, unconsciousness, and neck stiffness, with a negative SRAS-CoV2 RNA nasopharyngeal swab, but positive in cerebrospinal fluid (CSF); a magnetic resonance image (MRI) showed hyperintensities in the wall of the right lateral ventricle, right mesial temporal and hippocampus, suggesting the diagnosis of meningoencephalitis associated with SARS-CoV2 [9]. In another patient with altered mental status, SARS-CoV2 RNA was detected in a nasopharyngeal swab specimen; the MRI demonstrated hemorrhagic, rim-enhancing lesions, within both thalami, medial temporal lobes, and subinsular regions, establishing the diagnosis of acute necrotizing encephalopathy associated with COVID-19 [10]. A different patient with acute flaccid lower extremities paralysis and urinary and bowel incontinence after a fever spike was positive for SARS-CoV2 in a nasopharyngeal swab; this clinical picture suggests a direct virus invasion to the ACE2 receptors of the spinal cord cells, causing acute myelitis [11].

It is of paramount importance not to forget the possible comorbidity between neurologic diseases and COVID-19. Patients with autoimmune diseases such as multiple sclerosis, myasthenia gravis, neuromyelitis optica or sarcoidosis are under therapy with immunosuppressive drugs, with a subsequence higher risk of getting COVID-19. Extra precautions should be taken with these patients, both in the preventive measures to avoid getting the infection and with the possible adjustment of the medications for their diseases.

The current pandemi c took us all by surprise because the cause is a microorganism previously unknown, so the scientific knowledge and evidence about both symptoms and acute and chronic possible complications that the SARS-CoV2 can cause and the possible interactions with the nervous system or the treatments used in neurological disorders are being built. For this reason, it is essential that all physicians do not forget to take all these aspects into account when evaluating their patients and to communicate the knowledge acquired from interaction with COVID-19 cases. This is the only way in which we can provide help to all the physicians that are currently in the frontline, preventing them from making errors, and subsequently, may we expect so, reducing the number of active cases and deaths that this disease is bringing upon us.

\section{REFERENCES}

1. Mao L, Wang $M$, Chen S, He Q, Chang J, Hong C, Hu B. Neurological Manifestations of Hospitalized Patients with COVID-19 in Wuhan, China: A Retrospective Case Series Study. Lancet Neurol. 2020. https://doi.org/10.2139/ssrn.3544840

2. Jin H, Hong C, Chen S, Zhou Y, Wang Y, Mao L, Hu B. Consensus for prevention and management of coronavirus disease 2019 (COVID-19) for neurologists. Stroke Vascular Neurol. 2020. https://doi.org/10.1136/svn-2020-000382

3. Gautier JF, Ravussin Y. A New Symptom of COVID-19: Loss of Taste and Smell. Obesity (Silver Spring, Md.); 2020. https://doi.org/10.1002/oby.22809

4. Giacomelli A, Pezzati L, Conti F, Bernacchia D, Siano M, Oreni L, Galli M. Self-reported olfactory and taste disorders in SARS-CoV-2 patients: a crosssectional study. Clinical Infect Dis. 2020;53(9):1689$1699 . \quad$ https://doi.org/10.1093/cid/ciaa330

5. Brann D, Tsukahara T, Weinreb C, Logan DW, Datta SR. Non-neural expression of SARS-CoV-2 entry genes in the olfactory epithelium suggests mechanisms underlying anosmia in COVID-19 patients. BioRxiv. 2020.

6. Baig AM, Khaleeq A, Ali U, Syeda H. Evidence of the COVID-19 Virus Targeting the CNS: Tissue Distribution, Host-Virus Interaction, and Proposed Neurotropic Mechanisms. ACS Chemical Neurosc. 2020. https://doi.org/10.1021/acschemneuro.0c00122

7. Li YC, Bai WZ, Hashikawa T. The neuroinvasive potential of SARS-CoV2 may be at least partially responsible for the respiratory failure of COVID-19 patients. J Med Virol. 2020, 2. https://doi.org/10.1002/jmv.25728 
8. Zhang $Y$, Xiao $M$, Zhang $S$, Xia $P$, Cao $W$, Jiang W, Zhang S. Coagulopathy and Antiphospholipid Antibodies in Patients with Covid-19. New England J Med. 2020. https://doi.org/10.1056/NEJMc2007575

9. Moriguchi T, Harii N, Goto J, Harada D, Sugawara H, TakaminoJ, ShimadaS. AfirstCaseofMeningitis/Encephalitis associated with SARS-Coronavirus-2. Int J Infectious Dis. 2020. https://doi.org/10.1016/j.ijid.2020.03.062

10. PoyiadjiN, Shahin G, NoujaimD, StoneM, PatelS, Griffith B. COVID-19-associated Acute Hemorrhagic Necrotizing Encephalopathy: CT and MRI Features. Radiology. 2020. https://doi.org/10.1148/radiol.2020201187

11. Zhao K, Huang J, Dai D, Feng $Y$, Liu L, Nie S. Acute myelitis after SARS-CoV-2 infection: a case report. MedRxiv. 2020. https://doi.org/10.1101/2020.03.16.20035105v1 